\title{
Detection of sentinel lymph nodes by SPECT/ CT and planar scintigraphy: The influence of age, gender and BMI
}

\author{
Otakar Kraft ${ }^{1,2}$, Martin Havel ${ }^{1}$ \\ 1. Clinic of Nuclear Medicine, University Hospital Ostrava, Ostrava, Czech Republic. 2. Faculty of Medicine, University of \\ Ostrava, Ostrava, Czech Republic.
}

Correspondence: Otakar Kraft. Address: University Hospital Ostrava, 17.listopadu 1790, 70852 Ostrava-Poruba, Czech Republic. Telephone: 42-059-737-2290. Email: otakar.kraft@centrum.cz

Received: May 15, 2012

Accepted: July 5, 2012

Published: December 1, 2012

DOI : $10.5430 / j b g c . v 2 n 2 p 11$

URL: http://dx.doi.org/10.5430/jbgc.v2n2p11

\section{Abstract}

Background: The most important prognostic factor in melanomas, breast cancers and gynecologic tumors is the state of the lymph nodes (LNs). There is not suitable pre-surgery examination procedure of detection of impacted LNs. The use of lymphatic mapping of sentinel lymph node (SLN) helps reduce the morbidity of surgery. Accurate visualization of the SLN is required for the best results. The aim is to assess the role of planar lymphoscintigraphy and fusion imaging of SPECT/CT in SLN detection in patients with various types of tumors and estimation of some factors influencing detection success - age, gender and body mass index (BMI).

Methods: Planar scintigraphy and hybrid modality SPECT/CT were performed in 550 patients (pts) (mean age $58.1 \pm 13.1$ years): 69 pts with gynecological tumors (37 with cervical cancer, 25 pts with endometrial cancer, 7 pts with vulvar carcinoma); in 161 pts with melanomas; and in 318 women and 2 men with breast cancer. The radiopharmaceutical was injected around the tumor, subareolar or around the scar. Planar and SPECT/CT images were interpreted separately by two nuclear medicine physicians. Efficacy of these two techniques to image SLN were compared. We have estimated the influence of the age, gender and BMI on the succes rate of SLN detection. Student's paired $t$-test was used for comparing numbers of found SLN by both techniques; F-test, Student's $t$-test, and Fisher's exact test were utilized for age, gender and BMI characteristics comparisons. Values were considered significant when $P<0.05$.

Results: Planar scintigraphy did not image SLN in 77 pts in total (14.0\%); in 8 pts with gynecologic tumors, in 23 pts with melanomas and in 46 pts with breast cancer. SPECT/CT was negative in 49 pts $(8.9 \%)$ in total; in 4 pts with gynecologic tumors, in 12 pts with melanomas and in 33 pts with breast cancer. In $199(36.2 \%)$ pts the number of SLNs captured on SPECT/CT was higher than on planar imaging. 35 foci of uptake (3.1\% from totally visible 1134 foci on planar images) interpreted on planar images as hot LNs were found to be false positive non-nodal sites of uptake when further assessed on SPECT/CT. Influence of the age, gender and BMI: The group of patients with higher number of detected SLN on SPECT/CT than on planar scintigraphy had lower average age than the group of patients with the same number of detected SLN on SPECT/CT and on planar scintigraphy, the difference was statistically significant $(P=0.008)$. BMI did not differ in these two groups. There has been proportionally higher number of men than women who have had higher detected SLNs on SPECT/CT than on planar scintigrams (difference statistically significant $P=0.04$ ). It follows that gender influences the difference of detected SLNs on SPECT/CT and planar scintigraphy in favor of male gender. 
Conclusion: In some patients with gynecologic, including breast cancers and melanomas SPECT/CT improves detection of SLNs as compared to planar scintigraphy. It can image nodes not visible on planar scintigrams, excludes false positive uptake and it improves anatomical localization of SLNs. We have found the influence of the age and gender on the difference of detected numbers of SLNs by the fusion of SPECT/CT and planar lymphoscintigraphy. This difference was not influenced by BMI.

\section{Key words}

Sentinel lymph node, Malignant tumors, Lymphoscintigraphy, Planar scintigraphy, SPECT/CT

\section{I ntroduction}

Sentinel lymph node (SLN) was defined as the first lymph node (LN) draining the primary tumor, i. e. the first LN that is at risk from metastatic cells ${ }^{[1]}$. The histological status of the SLN has been found to be an indicator representative of the whole LN basin. It has turned out to be the strongest prognostic factor for tumor recurrence and survival ${ }^{[2-6]}$, and a major decision criterion for adjuvant therapy ${ }^{[7,8]}$.

There is not suitable pre-surgery examination procedure of detection of impacted LNs. SLN biopsy is the most accurate and the only reliable method for nodal staging which can diagnose microscopic tumor spread to the regional LNs. Lymphoscintigraphy visualizes where lymph from the primary tumor site travels and is, therefore, an essential element of lymphatic mapping ${ }^{[9-11]}$. The most important benefits of the SLN procedure are avoidance of over treatment and prevention of morbidity ${ }^{[12-15]}$.

The use of lymphatic mapping of SLN may help reduce the morbidity of surgery without compromising the identification of higher-risk patients who require adjuvant treatment. The SLN protocol of enhanced pathologic evaluation of removed nodes may also provide a much more detailed evaluation of these nodes and the potential for identifying micrometastasis that may have been missed with traditional pathologic evaluation ${ }^{[16]}$. Accurate visualization of the SLN is required for the best results. The better anatomic definition and improved resolution that characterize SPECT images may overcome limitations of planar images. However, localization of hot LNs on SPECT images without anatomic landmarks is not possible. A decade ago, hybrid imaging combining fusion of single-photon emission tomography (SPECT) with computed tomography (CT) was introduced for simultaneously acquiring functional (physiological) and anatomical (morphological) information ${ }^{[17-19]}$. SPECT/CT was introduced in lymphatic mapping with the goal to show more SLNs and to show them more clearly than is possible with planar lymphoscintigraphy to improve nodal staging ${ }^{[20]}$. Hybrid SPECT/CT provides better contrast and resolution than planar imaging with possibility to correct an attenuation and scatter ${ }^{[21,22]}$. SPECT/CT images provide the topographic landmarks that may further facilitate surgical exploration ${ }^{[19]}$ with improvement of surgical SLNs detection. If only used to correct the radionuclide image for photon attenuation, the CT data can be acquired with a considerably lower statistical quality and coarser spatial resolution than required for diagnostic-quality imaging and therefore can deliver a significantly lower radiation dose than that for a diagnostic CT study ${ }^{[23]}$.

SLN has been incorporated in the routine management of various solid tumor types ${ }^{[24-26]}$. SLN biopsy has an established role in malignant melanoma and breast cancer ${ }^{[26,27]}$. A group of patients who might benefit from a SLN biopsy are those with cancer of the uterine cervix and other gynecological malignancies.

Minimal invasive SLN biopsy can replace lymphadenectomy for staging. Planar lymphoscintigraphic imaging is an important element in lymphatic mapping but interpretation of planar lymphoscintigrams is hindered by the absence of anatomical landmarks in the scintigraphy image ${ }^{[28]}$. An essential step in the procedure for SLN biopsy is to locate the first-echelon node of draining basin. In some patients, however, the lymphoscintigram shows a drainage pattern that is difficult to interpret and in a small minority no SLN is depicted at all ${ }^{[29]}$. 
In our study we compare hybrid SPECT/CT and planar lymphoscintigraphy in patients with gynecologic tumors, breast cancers, and melanomas exploring the value of SPECT/CT - mainly for detection of additional SLNs, the exact anatomical localization of SLNs, differentiation of skin contamination and estimation of the influence of age, gender and body mass index (BMI). SLN mapping is not always specific and work for increasing its specificity is needed. That is why we have studied possible associations of the results with age, gender and BMI.

\section{Materials, patients and methods}

Our study was approved by the Ethics Committee of the University Hospital of Ostrava. Informed consent was obtained from all patients.

\subsection{Patient population}

Planar and hybrid SPECT/low-dose CT lymphoscintigraphy were performed in 550 consecutive patients (mean age 58.1 \pm 13.1 years, BMI 27.8 \pm 5.1 ): 69 pts with gynecological tumors (37 with cervical cancer, 25 pts with endometrial

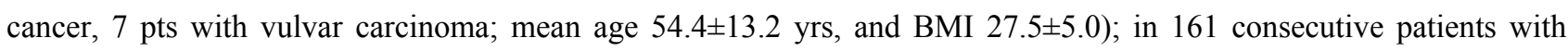
melanomas ( 87 men, 74 women, mean age 57.1 \pm 14.8 years, BMI 28.4 \pm 5.1 ); and in consecutive 318 women and 2 men with breast cancer (mean age 59.4 \pm 12.0 yrs, BMI 27.6 \pm 5.1 ) with no clinical evidence of LN metastases (N0) and no remote metastases (M0).

\subsection{Lymphoscintigraphic method}

In patients with gynecologic tumors (GROUP A), we performed preoperative lymphoscintigraphy utilizing 99mTc-colloid, activity $40 \mathrm{MBq}$, on the operation day (one-day protocol). Gynecologists injected 4 peritumoral injections of colloid around the tumor. Scintigraphy followed 25-60 minutes after injection. In melanoma patients (GROUP B) on the day of surgery (one day protocol) an activity of $100 \mathrm{MBq} 99 \mathrm{mTc}$ labelled colloid divided in four equal aliqouts of 0.5 $\mathrm{ml}$ was injected by nuclear medicine physician in four intradermal injection around the tumor (79 pts) or around the scar (82 pts) after excision of the melanoma. In breast cancer patients (GROUP C) on the day prior to surgery (2 day protocol in 273 patients) or on the day of surgery (one day protocol in 47 women) an activity of $120 \mathrm{MBq}$ of 99mTc labelled colloid divided in four or five equal aliqouts of $0.5 \mathrm{ml}$ was injected by nuclear medicine physician. Women with a palpable mass were injected in four peritumoral sites and one subdermal injection above the tumor ( $273 \mathrm{pts})$. Patients with nonpalpable tumor were injected in four subareolar sites (47 pts). We have used these $99 \mathrm{mTc}$ - colloids: Nanocis (size of colloid particles $100 \mathrm{~nm}-68 \mathrm{pts}$ in all groups), Nanocoll (size of colloid particles to $80 \mathrm{~nm}-379 \mathrm{pts}$ ), SentiScint (size of particles to $200 \mathrm{~nm}-5 \mathrm{pts}$ ), NanoAlbumon (size of particles to $80 \mathrm{~nm}-98 \mathrm{pts}$ ). Choice of radiopharmaceuticals (Rf) was totally random. Type of Rf was not important because we have compared planar scintigraphy and SPECT/CT performed by the same Rf in individual patient.

In melanoma patients in the first the dynamic scintigraphy (parameters: matrix $128 \times 128,30$ frames, 20 s per frame) was done. In all types of tumors the planar scintigraphy (in melanoma patients planar scintigraphy was done immediately after dynamic scintigraphy) and SPECT/CT lymphoscintigraphy was performed using a hybrid system composed of a dual-head gamma camera with a low-dose CT installed in a gantry (Symbia T2 Siemens).

Planar lymphoscintigraphy was carried out in the anterior, posterior, lateral and in some patients oblique projections focusing on the area of interest. Acquisition time was 10 minutes. If SLN was displayed, to facilitate the surgical resection a reference mark was placed on the skin with the help of the 57Co mark, corresponding to the position of the SLN visualized by lymphoscintigraphy. SPECT/CT images were acquired immediately after planar images. The SPECT/CT system (Symbia T2; Siemens, Erlangen, Germany) consisted of a dual-head variable-angle gamma camera equipped with low-energy high-resolution collimators and a two-slice spiral CT scanner optimized for rapid rotation. SPECT acquisition (matrix 128 $\times 128,60$ frames at 25s per view) was performed using steps of $6^{0}$. CT scan was a low-dose, noncontrast study 
(130 kV, $17 \mathrm{mAs}$, B60s kernel), 5-mm slices were created. The iterative reconstruction (OSEM 3D) was used for generating SPECT slices. Both SPECT images and CT axial slices were fused using an Esoft 2000 application package (Siemens, Erlangen, Germany). Hybrid SPECT/CT images were viewed using two-dimensional orthogonal re-slicing in axial, sagittal and coronal orientation. Maximum intensity projections with a three-dimensional display were generated to localize SLNs in relation to anatomical structures.

\subsection{Scintigraphic interpretation}

SLN localization was interpreted separately on planar and SPECT/CT images. Image analysis was performed prospectively by two experienced nuclear medicine physicians in consensus reading. All images were analyzed in two steps: 1. analysis of planar images; 2. analysis of coregistered SPECT/CT images. The location of SLNs was categorized as axillar, inguinal, pelvic, paraaortic and in other lymphatic basins.

In the analysis of the results, fused SPECT/CT images data were concluded to be clinically relevant if they identified SLNs which were missed on planar images of a specific basin, if they excluded SLN suspected on planar images, or if they localized SLNs in additional or different basins than those suggested by planar images.

\subsection{Statistical test}

Student's paired $t$-test was used for comparing numbers of found nodes by both techniques; F-test, Student's $t$-test, and Fisher's exact test were utilized for age, gender and BMI characteristics comparisons. Values were considered significant when $P<0.05$.

\subsection{Surgery}

On the basis of the scintigraphic findings on both planar and SPECT/CT images the surgeon or gynecologist is guided during surgery. An intraoperative hand-held probe (NEO 2000, Neoprobe Corporation Dublin, Ohio, USA; detector: crystal of Cadmium Zinc Telluride; $12 \mathrm{~mm}$ collimated angulated probe) has been used in all patients before incision to identify the site with the highest counts of LNs in the lymphatic basin. In some patients a patent blue dye (BLEU PATENTÉ V 2.5\%, Guebert, France) has been injected similarly to the earlier colloid injection.

The surgeon removed all detected SLNs excluding parasternal (intramammary chain - IMC) nodes, which are not routinely harvested. If some of SLNs in patients with breast cancer was metastatic, axillary dissection followed (in the same day or later).

\section{Results}

On SPECT/CT images, hot nodes in 501 of the 550 (91.1\%) study patients were detected, with a mean of $2.9 \pm 2.9$ (range 1-11) nodes per patient. SPECT/CT showed the exact anatomical location of all visualized SLNs. There was failure to detect SLNs in the remaining 49 (8.9\%) patients. Planar images identified SLNs in 473 (86.0 \%) patients, with a mean of $2.4 \pm 1.7$ (range 1-12 nodes) per patient. In the remaining 77 (14.0\%) patients no SLNs were detected on planar images.

343 LNs in 199 (36.2 \%) patients were missed on planar images, but identified on SPECT/CT. Plus, 35 (3.1 \%) foci of uptake in 29 patients interpreted on planar images as hot LNs were found to be false positive non-nodal sites of uptake when further assessed on SPECT/CT. In 31 (5.6 \%) patients, who had planar imaging negative, SPECT/CT visualized lymphatic drainage.

In GROUP A (pts with gynecologic tumors), planar lymphoscintigraphy alone visualized SLN in 61 (11.1\%) patients as compared to SPECT/CT imaging that identified SLN in 65 patients $(11.8 \%)$. The number of SLNs captured on 
SPECT/CT was higher than on planar imaging in 38 (55.1\%) patients $(P<0.001)$. In 4 (5.8 \%) patients, who had planar imaging negative, SPECT/CT visualized lymphatic drainage.

In GROUP B (pts with melanoma), the SLN detection rate by SPECT-CT lymphoscintigraphy was $92.5 \%$ (in 149 patients), by planar imaging $85.7 \%$ (in 138 patients), respectively $(P<0.001)$. In 12 patients ( $7.5 \%)$ SPECT-CT failed to detect hot SLNs. In 12 patients (7.5\%), SLNs were detected only by SPECT-CT and there was no patient with SLN detected only by planar images. Hybrid imaging brought additional value especially in the group of patients with scar after extirpation of melanoma, where SPECT/CT visualized higher number of SLNs in $20 \%$ more cases than in the group of patients prior the primary surgery.

The overall SLN detection rate on SPECT-CT imaging in GROUP C (pts with breast cancer) was $89.7 \%$ (in $287 \mathrm{pts}$ ), and $85.5 \%$ (in 274 pts) by planar scintigraphy $(P<0.001)$. In 33 patients $(10.3 \%)$ SPECT-CT imaging failed to detect hot SLNs. In 15 (4.7\%) patients, SLNs were detected only on SPECT/CT images.

We found the number of LNs detected on SPECT-CT significantly higher than on planar scintigraphy $(P<0.001)$ in all 550 patients and in individual three groups (A, B and C), too. In 98 pts examined by Nanoalbumon, in 68 pts examined by Nanocis and in 379 pts examined by Nanocoll the number of LNs detected on SPECT-CT was significantly higher than on planar scintigraphy (for Nanoalbumon $P<0.001$, for Nanocis $P=0.002$, for Nanocoll $P<0.001$ ). Small number of patients (5 pts) examined by Sentiscint was not analyzed separately, but only in scope of all 550 patients. Numbers of found lymphnodes in each group for each method are mentioned in Table 1.

Table 1. Numbers of detected lymphnodes

\begin{tabular}{lllll} 
& GROUP A & GROUP B & GROUP C & Total \\
\hline Planar & 187 & 351 & 596 & 1134 \\
SPECT/CT & 263 & 487 & 692 & 1442 \\
\hline
\end{tabular}

The age, gender and BMI influencing detection rate by planar scintigraphy and fusion of SPECT/CT:

The group of patients with higher number of detected SLN on SPECT/CT than on planar scintigraphy (199 pts) has had a lower average age ( $56.4 \pm 13.8$ years) than the group of patients (351 pts) with the same number of detected SLN on SPECT/CT and on planar scintigraphy $(59.2 \pm 12.6$ years $)$, the difference is statistically significant $(P=0.008)$.

BMI did not differ in these two groups (average BMI in the group of patients with higher number of detected SLN on SPECT/CT than on planar scintigraphy was $27.7 \pm 5.1$; average BMI in the group of patients with the same number of detected SLN on SPECT/CT and on planar scintigraphy was $27.9 \pm 5.1)$. The difference was not statistically significant $(P=0.370)$, in group A $P=0.3835$, in group B $P=0.2144$, in group C $P=0.1059$.

To confirm the influence of age we have compared two groups of patients with age higher than 65 years (179 pts) and with age equal or lower than 65 years (371). In the group of the older patients there is lower yield of SPECT/CT than in the younger patients. Difference is statistically significant $(P=0.0159)$ - Table 1 (explanation: SPECT/CT $>$ planar - higher number of detected SLNs on SPECT/CT than on planar scintigraphy; SPECT = planar - the same number of detected SLNs on SPECT/CT as on planar scintigraphy).

Further we have compared two groups of patients according to the gender - men (87 with melanoma and 2 with breast cancer) and women (461 from all three groups). There has been proportionally higher number of men than women who have had higher detected SLNs on SPECT/CT than on planar scintigrams. Difference is statistically significant $(P=0.040)$ - Table 2 (explanation: SPECT/CT > planar - higher number of detected SLNs on SPECT/CT than on planar scintigraphy; SPECT $=$ planar - the same number of detected SLNs on SPECT/CT as on planar scintigraphy). 
Table 2. Influence of the age on SLN detection on SPECT/CT

\begin{tabular}{llll}
\hline In all groups & SPECT/CT $>$ planar & SPECT $=$ planar \\
\hline Pts $>65$ yrs & 53 & 126 \\
Pts $\leq 65$ yrs & 146 & 225 & $P=0.0159$ \\
\hline
\end{tabular}

It follows that gender influences the difference of detected SLNs on SPECT/CT and planar scintigraphy in favor of male gender.

Table 3. Influence of the gender on SLN detection on SPECT/CT

\begin{tabular}{llll}
\hline In all groups & SPECT $/$ CT $>$ planar & SPECT $=$ planar \\
\hline Men & 40 & 49 & \\
Women & 159 & 302 & \\
& & & $P=0.040$ \\
\hline
\end{tabular}

\section{Discussion}

Low radiation dose is added to the scintigraphic mapping by the low-dose CT, ranging from $1.3 \mathrm{mGy}$ at the centre to 5 $\mathrm{mGy}$ at the surface of the body ${ }^{[19,30]}$. According to low-dose scanning protocol we have evaluated the radiation exposure from spiral CT to about 1.5 millisieverts (mSv). Hybrid system allows transmission (low-dose CT) and emission (SPECT) scans to be performed without changing the patient's position, thereby allowing for automatic and correct record of images obtained with two modalities. It does not need additional manipulation with patient, so the total acquisition time is not prolonged considerably.

By visualizing SLN location and neighbouring structures by SPECT/CT information, less invasive treatment and thus a reduction of operative time, blood loss, and morbidity is likely to occur ${ }^{[17]}$. SPECT/CT has also been reported to reduce the false-positive interpretation of radiotracer accumulation in the event of contamination or radiotracer in lymphatic vessels ${ }^{[31]}$. One of the limitations of planar imaging in the detection of SLN is its inability to distinguish nodes that are superimposed ${ }^{[32]}$. A node close to the injection site can also be masked as the result of strong activity from the injection site (the "shine effect"), oblique projection can help ${ }^{[33]}$. 3-D SPECT/CT images may identify hidden nodes due to a good separation between counts related to the tracer injection and those of a closely located hot $\mathrm{LN}^{[34]}$. SPECT/CT is considered as gold standard and in our further study we will compare and validate our results by the surgery.

Contamination, nodes close to the injection site, and overweight patients are three noted instances in which SLN identification and localization are better with SPECT/CT than with standard planar methods. However, SLN mapping is not always specific and more work for increasing its specificity is needed. We have studied possible associations of the results with age, gender and BMI.

The better resolution of SPECT itself, the improved quality of attenuation-corrected SPECT images and the improved anatomical localization of nodes offered by the three-dimensional data of the SPECT reconstructed planes as well as the anatomical landmarks on CT may have contributed to the better nodal identification by SPECT/CT found in the study of Lerman et al. ${ }^{[35]}$. Higher nonvisualization of SLNs in overweight patients with breast cancer was described ${ }^{[36]}$. Lerman et al. ${ }^{[36]}$ stated that the rate of false negative planar imaging results for 122 overweight and obese patients was $28 \%$, higher than that for the general study population. The rate of false negative SPECT/CT results for these 122 patients was also higher than that for the general study population, $11 \%$; however, the latter modality identified hot nodes in 18 additional patients (53\%) and had a statistically higher rate of detection of SLNs in overweight patients. The addition of SPECT/CT to the acquisition protocol for lymphoscintigraphy in overweight and obese patients with breast cancer improves the 
identification of SLNs and avoids false-positive interpretations of sites of nonnodal uptake. In our study BMI did not influence the difference of detected SLNs on SPECT/CT and on planar scintigraphy in contrast with Lerman et al. ${ }^{[36]}$. For this finding we have no explanation.

Some authors have stated that SPECT/CT should only be performed in selected patients, i. e. those with an unusual lymphatic drainage pattern, with planar images that are difficult to interpret or with no visualization on planar images. In these patients, SPECT/CT appears to have additional value ${ }^{[37]}$. Ploeg et al. ${ }^{[37]}$ limited the use of SPECT/CT to difficult and unusual cases because they believe planar lymphoscintigraphy is an excellent preoperative mapping technique for most patients. They added nonvisualization as a new indication, because SPECT/CT visualized drainage in patients whose planar images did not reveal a SLN. They believe that the added costs and extra time for SPECT/CT are more justified when the procedure is used for this new indication. The present study confirms the additional value of SPECT/CT in the anatomical localization of SLNs and underlines its relevance for the surgical approach. SPECT/CT in our opinion, therefore, facilitates surgical exploration and may improve staging. We prefer performing SPECT/CT in all patients, not only in special situations. Other investigators have also concluded that additional SPECT/CT after planar lymphoscintigraphy resulted in an improved anatomical localization of SLNs. Neither in the present study nor in studies of other investigators SPECT/CT did miss SLN that was visualized by planar lymphoscintigraphy ${ }^{[20]}$. Whether SPECT/CT should be used in all patients or only for specific indications needs to be studied further ${ }^{[37]}$. It is reasonable to consider utilizing SPECT/CT in conjunction with planar imaging when feasible, especially in patients with negative planar imaging, in the hope of enhancing SLN detection and localization. If these data are confirmed, the routine use of SPECT in all patients may potentially have incremental value.

SPECT/CT may also obviate preoperative skin marking (we already have not done skin marking with time-saving) and may replace delayed lateral planar imaging.

In this study, nodes were accurately located to the anatomic sites. Accurate anatomic localization of SLN nodes preoperatively can also aid in probe-directed surgery and may reduce operator dependent variation and time. Preoperative SPECT/CT appears to enhance the topographic localization of SLN that may aid the surgeon in localizing SLN against surrounding structures.

According to our information from literature so far anybody has not ever evaluated the effect of age and gender on the difference of detection success of SLN using planar scintigraphy and SPECT/CT. The effect of the age was investigated using only planar scintigraphy by Birdwell et al. ${ }^{[38]}$ and by Brenot-Rossi et al. ${ }^{[39]}$. Both authors investigated influence of age only in women with breast cancer and came to opposite results. Brenot-Rossil et al. ${ }^{[39]}$ analyzed different parameters, such as the number of positive LNs, presence of lymphovascular invasion, tumor size, tumor grade, histology, prior excisional biopsy, and patient age to determine whether they had any significant correlation with nonvisualization of SLNs in the axillary area. Patient age at diagnosis was stratified as $<70$ or $>70$ years. An increased risk of unsuccessful axillary mapping was statistically associated with the number of positive axillary nodes. The age showed a statistical trend with lymphoscintigraphy failure: age $<70$ y old versus $>70$ y old $(P<0.096$, Fisher exact test $)$ - patient age showed no relation to unsuccessful lymphoscintigraphy. Birdwell et al. ${ }^{[38]}$ compared patients with visualized and nonvisualized SLNs for age, tumor site, size, and histologic findings, injection guidance method, diagnostic biopsy type, interval between biopsy and lymphoscintigraphy, intraoperative identification method, and surgical identification rate. They proved different finding than Brenot-Rossi et al. ${ }^{[39]}$ - there was a statistically significant difference between groups, with an age range of 29-79 years (mean age, 51.8 years) in patients with visualized SLNs and of 40-81 years (mean age, 60.2 years) in patients with nonvisualized SLNs. Patients with nonvisualized SLNs were older than those with visualized SLNs or by other words, patients with and those without visualization differed in age. This is similar to experience of $\mathrm{Ng}$ et al ${ }^{[40]}$, who reported an increase in failure to identify SLNs in women older than 50 years of age. 
We analyzed patients not only with breast cancers but also with other gynecologic tumors and melanoma. We have found that in people with lower age the higher average number of SLNs on SPECT/CT than on planar scintigraphy has been detected in the comparison with older patients. It may be related to a decrease in tissue turgor, with a resultant decrease in the hydrostatic intralymphatic pressure that drives the mapping agent into LN. Even if the agent is delivered successfully to the node, it may not be concentrated because of the limited sinusoidal space that remains in a fat-replaced node, another feature found more commonly in older patients ${ }^{[38]}$.

In our study, in melanoma patients the gender influenced the difference of detected SLNs on SPECT/CT and planar scintigraphy in favor of male gender. We can only speculate and argue why this is so. Possible influencing factors can be those: higher fat content with fatty degeneration of LNs in women compared with men and different physique of women.

On the other hand BMI did not differ between the group of patients with higher number of detected SLN on SPECT/CT than on planar scintigraphy and the group of patients with the same number of detected SLN on SPECT/CT and on planar scintigraphy.

Our study highlights the usefulness of SPECT imaging for SLN detection in breast cancers and other gynecologic tumors, and melanomas.

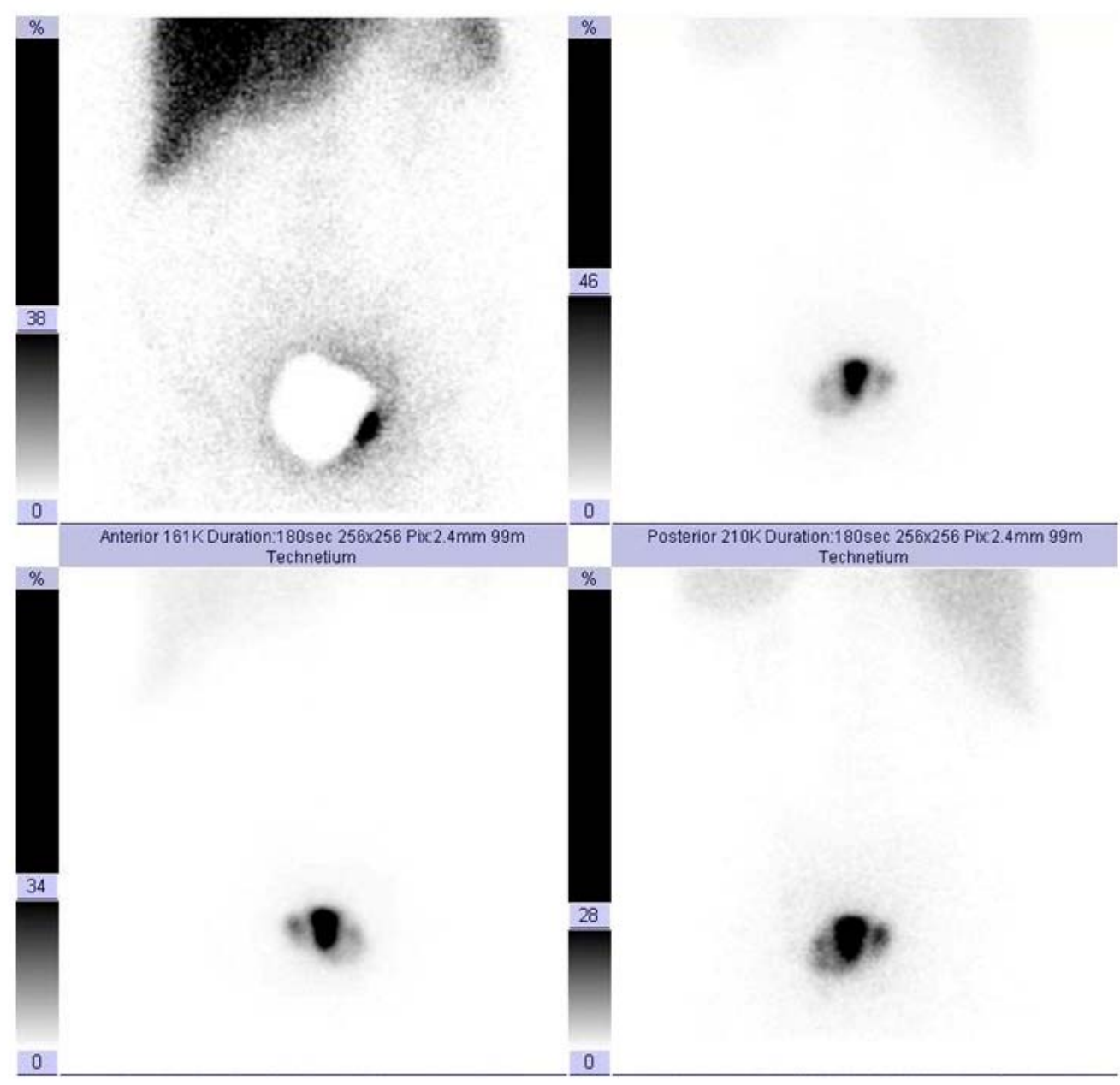

Figure 1. 54 years old woman with endometrial cancer. In the planar image in anterior and posterior projections no SLN is visible. 


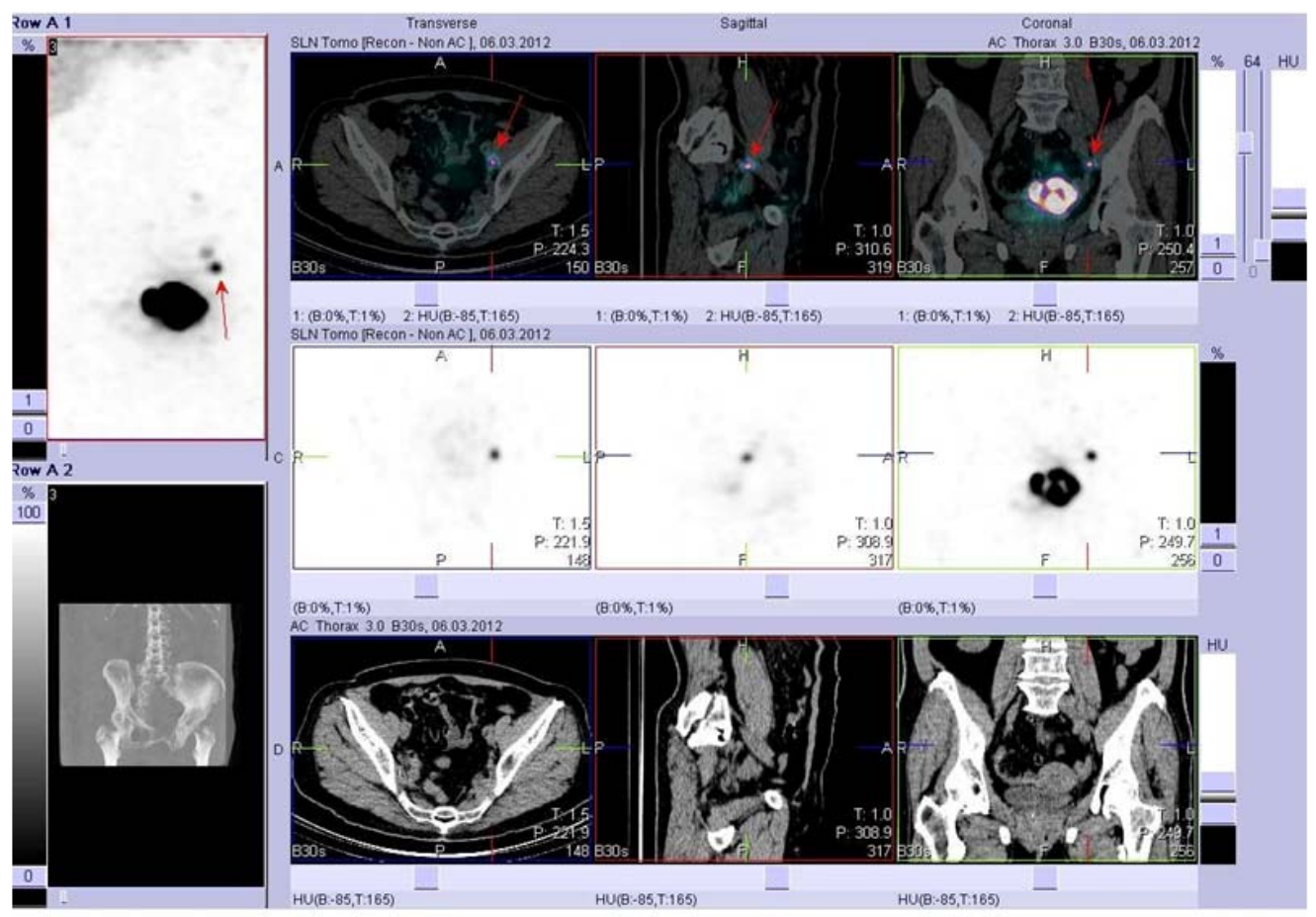

Figure 2. The same patient as in the figure 1. Fusion of SPECT and CT. Exact localization of SLN in the left part of pelvis.

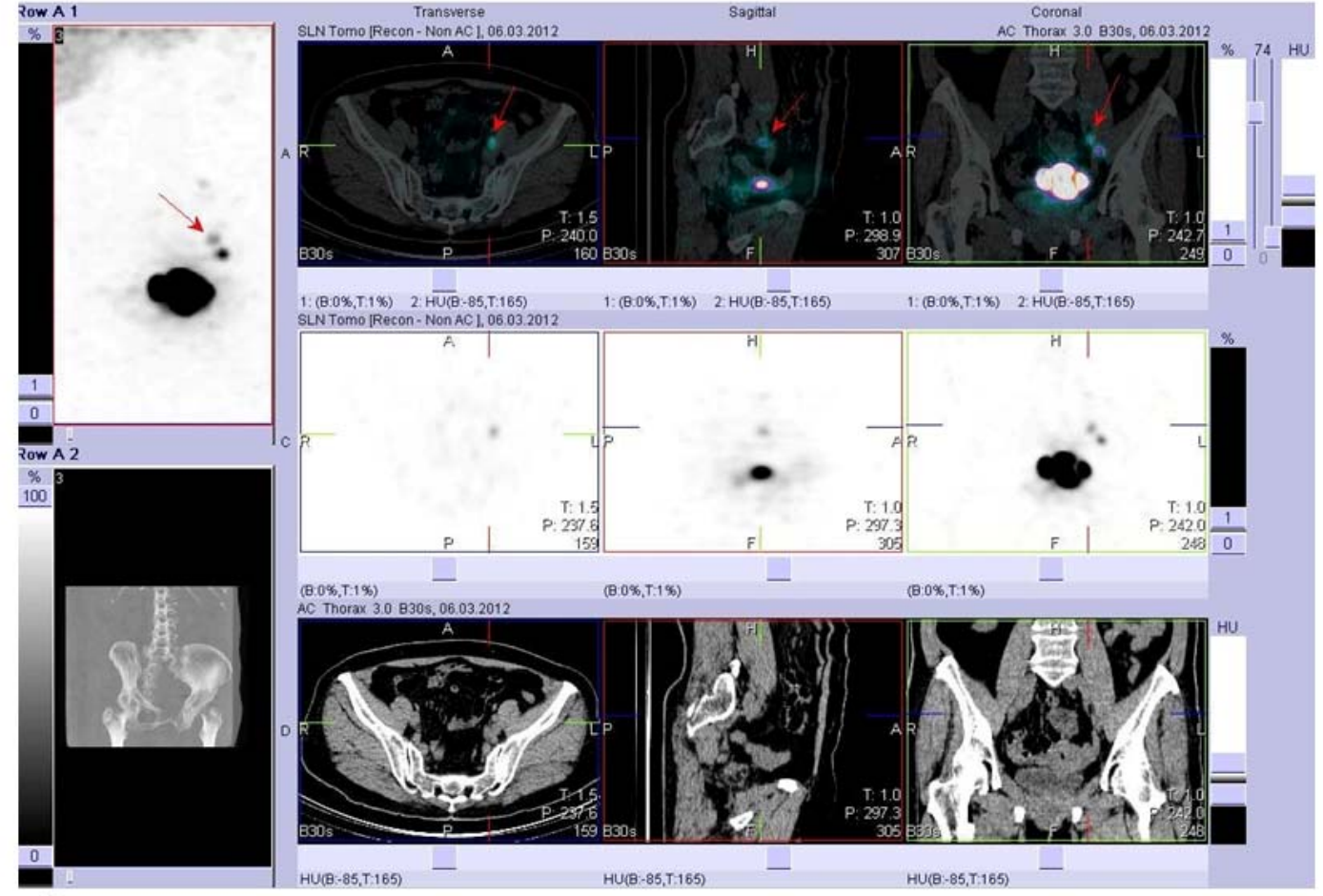

Figure 3. The same patient as in the figure 1 and 2. Fusion of SPECT and CT. Exact localization of the second SLN in the left part of pelvis. 


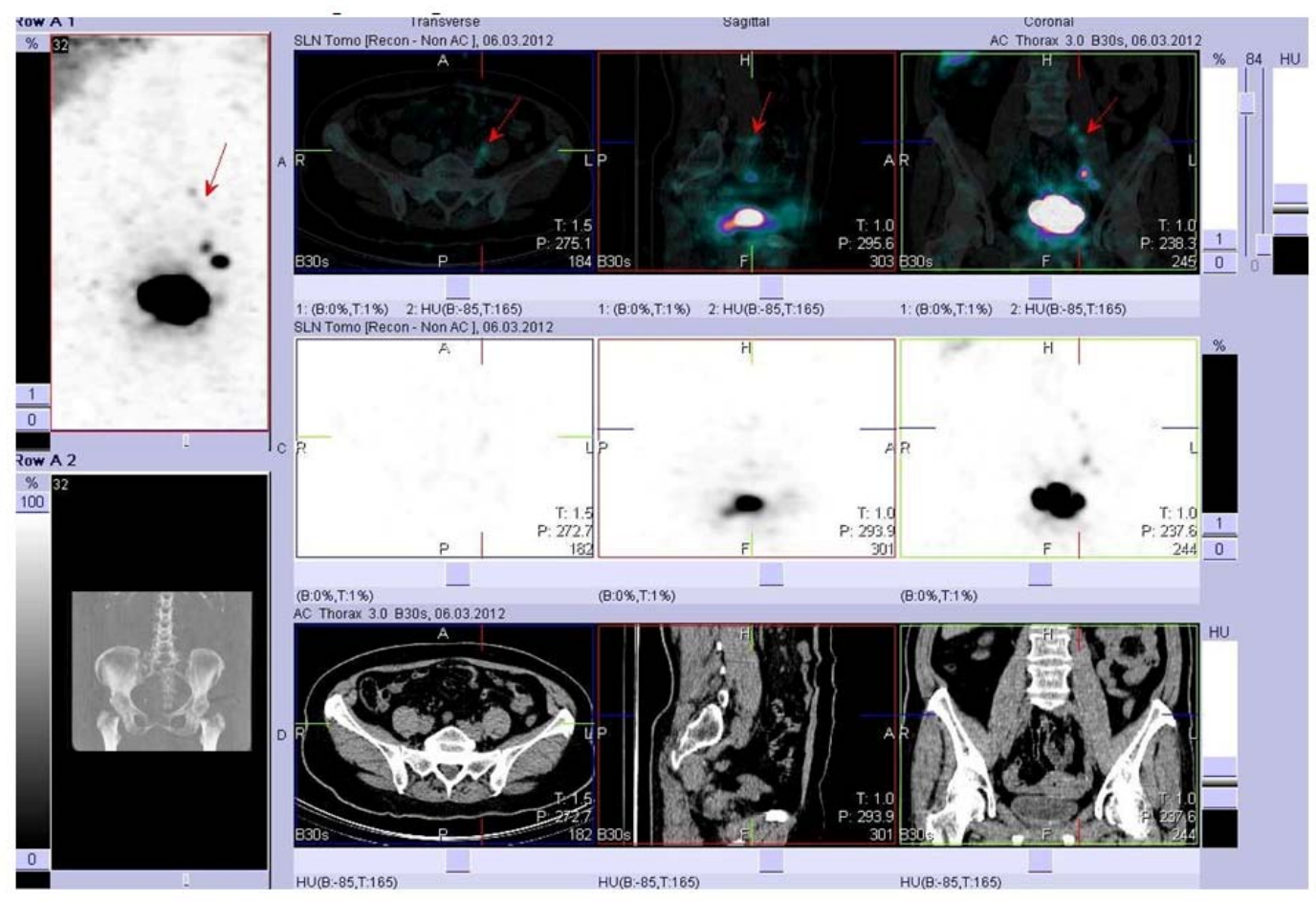

Figure 4. The same patient as in the figure 1,2 and 3. Fusion of SPECT and CT. Exact localization of the third SLN in the left part of pelvis.

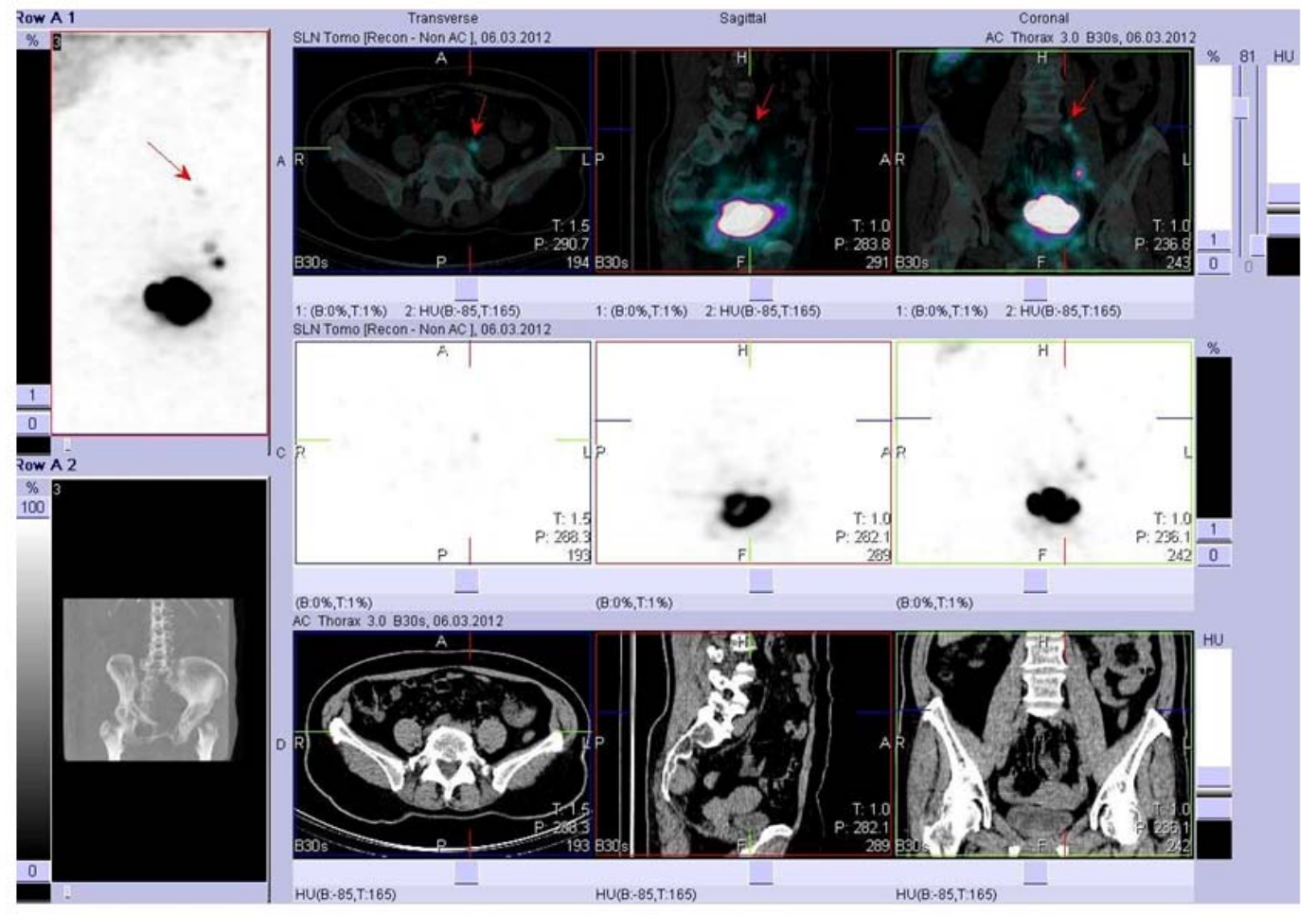

Figure 5. The same patient as in the figure 1,2,3 and 4. Fusion of SPECT and CT. Exact localization of the fourth SLN in the left part of pelvis 


\section{Conclusion}

The addition of SPECT/CT to planar lymphoscintigraphy improves the localization of preoperative draining nodes in patients with melanomas and breast or other gynecological cancers. It detects SLNs missed by planar imaging, excludes nonnodal false positive sites of uptake and accurately shows the exact anatomical location of visualized SLNs in patients with inconclusive conventional lymphoscintigrams.

Some factors, for example lower age and male gender, may influence imaging of SLNs with better results on SPECT/CT in comparison with older patients and women. However, BMI did not influence detection results on SPECT/CT in comparison with planar lymphoscintigraphy.

\section{References}

[1] Kretschmer L, Altenvoerde G, Meller J et al. Dynamic lymphoscintigraphy and image fusion of SPECT and pelvic CT-scans allow mapping of aberrant pelvic sentinel lymph nodes in malignant melanoma. Eur J Cancer. 2003; 39: 175-183. http://dx.doi.org/10.1016/S0959-8049(02)00534-8

[2] Reintgen D, Cruse CW, Wells K et al. The orderly progression of melanoma nodal metastases. Ann Surg. 1994; $220: 759-767$. PMid:7986143 http://dx.doi.org/10.1097/00000658-199412000-00009

[3] Kraft O, Ševčík L, Klát J et al. Detection of sentinel lymph nodes in cervical cancer. A comparison of two protocols. Nucl Med Rev Cent East Eur. 2006; 9: 65-68. PMid:16791808

[4] Klát J, Ševčík L, Šimetka O et al. Characteristics of sentinel lymph nodes' metastatic involvement in early stage of vulvar cancer. ANZJOG. 2009; 49: 672-676. PMid:20070721

[5] Jansen L, Nieweg OE, Peterse JL et al. Reliability of sentinel node biopsy for staging melanoma. Br J Surg. 2000; 87: 484-489. PMid:10759748 http://dx.doi.org/10.1046/j.1365-2168.2000.01362.x

[6] Lyman GH, Giuliano AE, Somerfield MR et al. Society of Clinical Oncology guideline recommendations for sentinel lymph node biopsy in early-stage breast cancer. J Clin Oncol. 2005; 23(30): 7703-7720. PMid:16157938 http://dx.doi.org/10.1200/JCO.2005.08.001

[7] Tanaka Y, Sawada S, Murata T. Relationship between lymph node metastases and prognosis in patients irradiated post-operatively for carcinoma of the uterine cervix. Acta Radiol. 1984; 23: 455-459. http://dx.doi.org/10.3109/02841868409136048

[8] Delgado G, Bundy B, Zaino R et al. Prospective surgical-pathological study of disease free interval in patients with stage IB squamous cell carcinoma of the cervix: a Gynecologic Oncology Group study. Gynecol Oncol. 1990; 38: 352-357. http://dx.doi.org/10.1016/0090-8258(90)90072-S

[9] Estourgie SH, Nieweg OE, Valdés Olmos RA et al. Review and evaluation of sentinel node procedures in 250 melanoma patients with a median follow-up of 6 years. Ann Surg Oncol. 2003; 10: 681-688. PMid:12839854 http://dx.doi.org/10.1245/ASO.2003.01.023

[10] Vuylsteke RJ, Van Leeuwen PA, Statius Muller MG et al. Clinical outcome of stage I/II melanoma patients after selective sentinel lymph node dissection: long-term follow-up results. J Clin Oncol. 2003; 21: 1057-1065. PMid:12637471 http://dx.doi.org/10.1200/JCO.2003.07.170

[11] Hubalewska-Dydejczyk A, Sowa-Staszczak A, Huszno B. Current application of sentinel lymph node lymphoscintigraphy to detect various cancer metastases. Hell J Nucl Med. 2006; 9(1): 5-9. PMid:16617387

[12] Reeves ME, Coit DG. Melanoma. A multidisciplinary approach for the general surgeon. Surg Clin North Am. 2000; 80: 581-601. http://dx.doi.org/10.1016/S0039-6109(05)70202-4

[13] Shrenk P, Rieger R, Shamiyeh A et al. Morbidity following sentinel lymph node biopsy versus axillary lymph node dissection for patients with breast carcinoma. Cancer. 2000; 88: 608-614. http://dx.doi.org/10.1002/(SICI)1097-0142(20000201)88:3<608::AID-CNCR17>3.0.CO;2-K

[14] Pijpers R, Buist MR, van Lingen A et al. The sentinel node in cervical cancer: scintigraphy and laparoscopic gamma probe-guided biopsy. Eur J Nucl Med Mol Imaging. 2004; 31: 1479-1486.

[15] Abu-Rustum NR, Alektiar K, Iasonos A et al. The incidence of symptomatic lower-extremity lymphedema following treatment of uterine corpus malignancies: a 12-year experience at Memorial Sloan-Kettering Cancer Center. Gynecol Oncol. 2006; 103: 714-718. PMid:16740298 http://dx.doi.org/10.1016/j.ygyno.2006.03.055 
[16] Pandit-Taskar N, Gemignani ML, Lyall A et al. Single photon emission computed tomography SPECT-CT improves sentinel node detection and localization in cervical and uterine malignancy. Gynecol Oncol. 2010; 117: 59-64. PMid:20117827 http://dx.doi.org/10.1016/j.ygyno.2009.12.021

[17] Martínez A, Zerdoud S, Mery E et al. Hybrid imaging by SPECT/CT for sentinel lymph node detection in patients with cancer of the uterine cervix. Gynecol Oncol. 2010; 119: 431-435. PMid:20822803 http://dx.doi.org/10.1016/j.ygyno.2010.08.001

[18] Even-Sapir E, Lerman H, Lievshitz G et al. Lymphoscintigraphy for sentinel node mapping using a hybrid SPECT/CT system. J Nucl Med. 2003; 44: 1413-1420. PMid:12960185

[19] Keidar Z, Israel O, Krausz Y. SPECT/CT in tumor imaging: technical aspects and clinical applications. Semin Nucl Med. 2003; 33: 205-218. PMid:12931322 http://dx.doi.org/10.1053/snuc.2003.127310

[20] van der Ploeg IM, Valdés Olmos RA, Kroon BB, Nieweg OE. The hybrid SPECT/CT as an additional lymphatic mapping tool in patients with breast cancer. World J Surg. 2008; 32: 1930-1934. PMid:18478289 http://dx.doi.org/10.1007/s00268-008-9618-5

[21] Bocher M, Balan A, Krausz Y et al. Gamma camera-mounted anatomical X-ray tomography: technology, system characteristics and first images. Eur J Nucl Med. 2000; 27: 619-627. http://dx.doi.org/10.1007/s002590050555

[22] Schillaci O. Hybrid SPECT/CT: a new era for SPECT imaging? Eur J Nucl Med Mol Imaging. 2005; 32 : 521-524. PMid:15747153 http://dx.doi.org/10.1007/s00259-005-1760-9

[23] Seo Y, Mari C, Hasegawa BH. Technological development and advances in single-photon emission computed tomography/ computed tomography. Semin Nucl Med. 2008; 38: 177-198. PMid:18396178 http://dx.doi.org/10.1053/j.semnuclmed.2008.01.001

[24] Burke TW, Levenback C, Tornos C et al. Intraabdominal lymphatic mapping to direct elective pelvic and paraaortic lymphadenectomy in women with high-risk endometrial cancer. Results of a pilot study. Gynecol Oncol. 1996; 62: 169-173. PMid:8751545 http://dx.doi.org/10.1006/gyno.1996.0211

[25] Nieweg OE, Jansen L, Valdés Olmos RA et al. Lymphatic mapping and sentinel lymph node biopsy in breast cancer. Eur J Nucl Med. 1999; 26: S11-16. PMid:10199927 http://dx.doi.org/10.1007/s002590050572

[26] Kraft O, Šafarčík K, Stępień A. Sentinel Lymph Node Detection and Biopsy in Breast Cancer and Malignant Melanoma. World J Nucl Med. 2004; 3: 26-32.

[27] Sergieva S, Goranov K, Piperkova E et al. Role of radioimmunoscintigraphy and SPET in the diagnosis of patients with malignant melanoma. Nucl Med Commun. 1994; 15: 168-172. PMid:8190407 http://dx.doi.org/10.1097/00006231-199403000-00009

[28] Dickinson RL, Erwin WD, Stevens DM et al. Hybrid modality fusion of planar scintigrams and CT topograms to localize sentinel lymph nodes in breast lymphoscintigraphy: Technical description and phantom studies. Int J Mol Imaging. 2011; $298: 102$.

[29] van der Ploeg IM, Nieweg OE, Kroon BB et al. The yield of SPECT/CT for anatomical lymphatic mapping in patients with breast cancer. Eur J Nucl Med Mol Imaging. 2009; 36: 903-909. PMid:19139879 http://dx.doi.org/10.1007/s00259-008-1050-4

[30] Lieber KA, Standiford SB, Kuvshinoff BW, Ota DM. Surgical management of aberrant sentinel lymph node drainage in cutaneous melanoma. Surgery. 1998; 124: 757-762. PMid:9780998 http://dx.doi.org/10.1067/msy.1998.90943

[31] Husarik DB, Steinert HC. Single-photon emission computed tomography/computed tomography for sentinel node mapping in breast cancer. Semin Nucl Med. 2007; 37: 29-33. PMid:17161037 http://dx.doi.org/10.1053/j.semnuclmed.2006.08.001

[32] Kobayashi K, Ramirez PT, Kim EE et al. Sentinel node mapping in vulvovaginal melanoma using SPECT/CT lymphoscintigraphy. Clin Nucl Med. 2009; 34: 859-861. PMid:20139817 http://dx.doi.org/10.1097/RLU.0b013e3181becdaf

[33] Khafif A, Schneebaum S, Fliss DM et al. Lymphoscintigraphy for sentinel node mapping using a hybrid single photon emission CT (SPECT)/CT system in oral cavity squamous cell carcinoma. Head Neck. 2006; 28: 874-879. http://dx.doi.org/10.1002/hed.20434

[34] Even-Sapir E. Sentinel node scintigraphic mapping using SPECT/CT. In: Israel O, Goldsmith S, eds. Hybrid SPECT/CT imaging in clinical practice. Taylor and Francis Group, New York. 2006; 121-139.

[35] Lerman H, Metser U, Lievshitz G et al. Lymphoscintigraphic sentinel node identification in patients with breast cancer: the role of SPECT-CT. Eur J Nucl Med Mol Imaging. 2006; 33: 329-337. PMid:16220303 http://dx.doi.org/10.1007/s00259-005-1927-4

[36] Lerman H, Lievshitz G, Zak O et al. Improved sentinel node identification by SPECT/CT in overweight patients with breast cancer. J Nucl Med. 2007; 48: 201-206. PMid:17268015

[37] van der Ploeg IMC, Valdés Olmos RA, Nieweg OE et al. The additional value of SPECT/CT in lymphatic mapping in breast cancer and melanoma. J Nucl Med. 2007; 48: 1756-1760. PMid:17942802 http://dx.doi.org/10.2967/jnumed.107.043372

[38] Birdwell RL, Smith KL, Betts BJ et al. Visualization at preoperative lymphoscintigraphy. Radiology. 2001 ; 220 : $47-53$. PMid:11425971

[39] Brenot-Rossi I, Houvenaeghel G, Jacquemier J et al. Nonvisualization of axillary sentinel node during lymphoscintigraphy: is there a pathologic significance in breast cancer? J Nucl Med. 2003; 44: 1232-1237. PMid:12902412

[40] Ng PC, Chua AC, Lannin DP, et al. Age and surgeon experience: the only significant factors contributing to sentinel node mapping failure in breast cancer (abstr). Breast Cancer Res Treat. 1999; 57: 27. 